\title{
Stagonosporopsis trachelii causes leaf spot on Ningpo Figwort (Scrophularia ningpoensis) in China
}

\author{
Chen Zhang ${ }^{1} \cdot$ Yulin $^{\text {Qian }}{ }^{1} \cdot$ Xinyue Zheng $^{1} \cdot$ Ying Zhou $^{1} \cdot$ Qin Xiong $^{1}$ (D) \\ Received: 17 October 2018 / Accepted: 18 April 2019 / Published online: 7 May 2019 \\ (C) Australasian Plant Pathology Society Inc. 2019
}

\begin{abstract}
Severe occurrence of a leaf spot disease was found on leaves of Ningpo Figwort (Scrophularia ningpoensis) in the SunYatsen Botanical Garden in Nanjing, China, during the summer of 2017. The morphological characteristics of the fungus were identical with those described for Stagonosporopsis trachelii. Molecular analysis of the ribosomal internal transcribed spacer (ITS) regions, partial beta-tubulin and actin gene regions confirmed the identity of the fungus as $S$. trachelii. Koch's postulates were completed for the same $S$. trachelii isolates, thereby confirming that the pathogen causes leaf spot on Ningpo Figwort (S. ningpoensis) in China. This is the first report of S. trachelii causing leaf spot on Ningpo figwort in China and the world.
\end{abstract}

Keywords Scrophulariaceae $\cdot$ Perennial plant $\cdot$ Morphological characterisation $\cdot$ Phylogenetic tree $\cdot$ Pathogenicity $\cdot$ China

Scrophularia ningpoensis, commonly known as the Ningpo figwort or Chinese figwort, is a perennial plant in the family Scrophulariaceae. The root of this plant has been widely used as traditional medicinal material for centuries in China ( $\mathrm{Li}$ et al. 1999). Ningpo figwort root, known as Xuanshen in Chinese, is harvested in autumn in Jiangsu province, Zhejiang province and neighbouring areas, then dried under sunlight for later use in the treatment of a form of tuberculosis as well as mental, nervous and gastrointestinal conditions. The root material has also been found to possess antibacterial, antiprotozoal, antitumor, anti-inflammatory, and hepatoprotective activities (Pasdaran et al. 2012; Li et al. 2009; Yang et al. 2011).

In June 2017, many small, oval- or circular- shaped light brown spots were observed on the leaves of most of the Ningpo figwort plants growing in the SunYatsen Botanical Garden in Nanjing, Jiangsu Province, China $\left(32.02^{\circ} \mathrm{N}, 118^{\circ}\right.$ E). These initial spots often turned a dark brown color, became enlarged and irregular in shape, and developed a grey-white sporulating necrotic center surrounded by a yellow-green halo (Fig. 1a-c). In the late stages of the infection, leaf chlorosis

Chen Zhang and Yulin Qian contributed equally to this work.

Qin Xiong

xiongqin@njfu.edu.cn

1 Co-Innovation Center for Sustainable Forestry in Southern China, College of Biology and the Environment, Nanjing Forestry University, Nanjing 210037, China occurred, resulting in the wilting of entire leaves. The disease incidence rates of plants in the garden and leaves per plant were approximately $87 \%$ and $60 \%$, respectively. The objective of this study was to identify the causal agent of the described leaf spot disease on Ningpo figwort plants in China.

Symptomatic leaves collected from ten independent figwort plants in the garden were surface sterilised with $75 \%$ ethanol for $1 \mathrm{~min}$. The leaves were then cut into small pieces (approximately $0.5 \mathrm{~cm}^{2}$ ), plated onto potato dextrose agar (PDA) medium containing rifampicin $(100 \mu \mathrm{g} / \mathrm{ml})$ and streptomycin sulfate $(100 \mu \mathrm{g} / \mathrm{ml})$, and incubated at $25{ }^{\circ} \mathrm{C}$ in the dark. After 3 days, ten fungal colonies that grew from the leaf tissue were sub-cultured on PDA. Pure cultures of the ten isolates were obtained using a single-spore isolation method described by Chilvers et al. (2014) with a slight modification as follows: a conidial suspension was diluted to $1 \times 10^{3} / \mathrm{ml}$ rather than $1 \times 10^{4} / \mathrm{ml}$, and the single spores were maintained on $10 \%$ V8 medium for fifteen days (Fig. 2g-h). Colonies on PDA were $58-68 \mathrm{~mm}$ in diameter after 7 days at $25^{\circ} \mathrm{C}$, with a growth rate of $2.5-5.0 \mathrm{~mm}$ per day in the early stages. Colonies on PDA or V8 medium were regular-margined, green to olive grey in surface color, and woolly to floccose in texture. They also produced white to olive grey aerial mycelia and were greenish-brown on the reverse (Fig. 2c-f). Fungal structures were examined under a Zeiss Axio Scope.A1 microscope equipped with a high-resolution Carl Zeiss AxioCam MRc5x microscopy camera, and at least 30 units were measured per structure. Fungal morphology was as follows: Pycnidia measured $120-160 \mu \mathrm{m}$ in diameter 

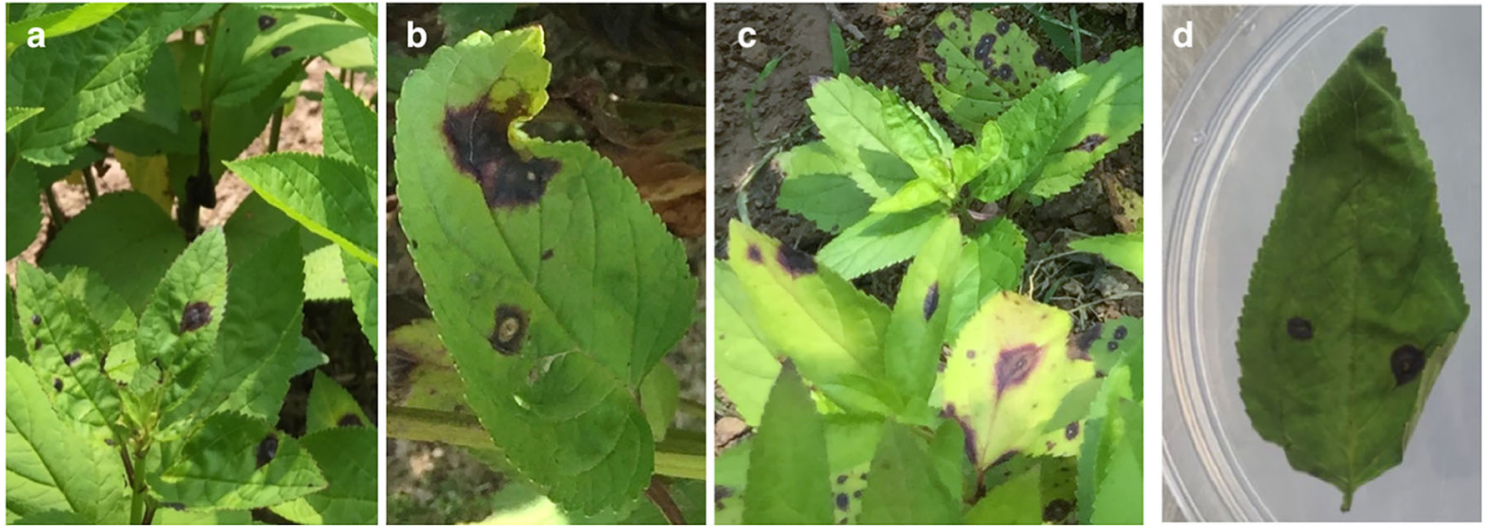

Fig. 1 Field symptoms (a-c) and artificial inoculated symptoms (d) of leaf spot on Ningpo figwort (Scrophularia ningpoensis) caused by Stagonosporopsis trachelii
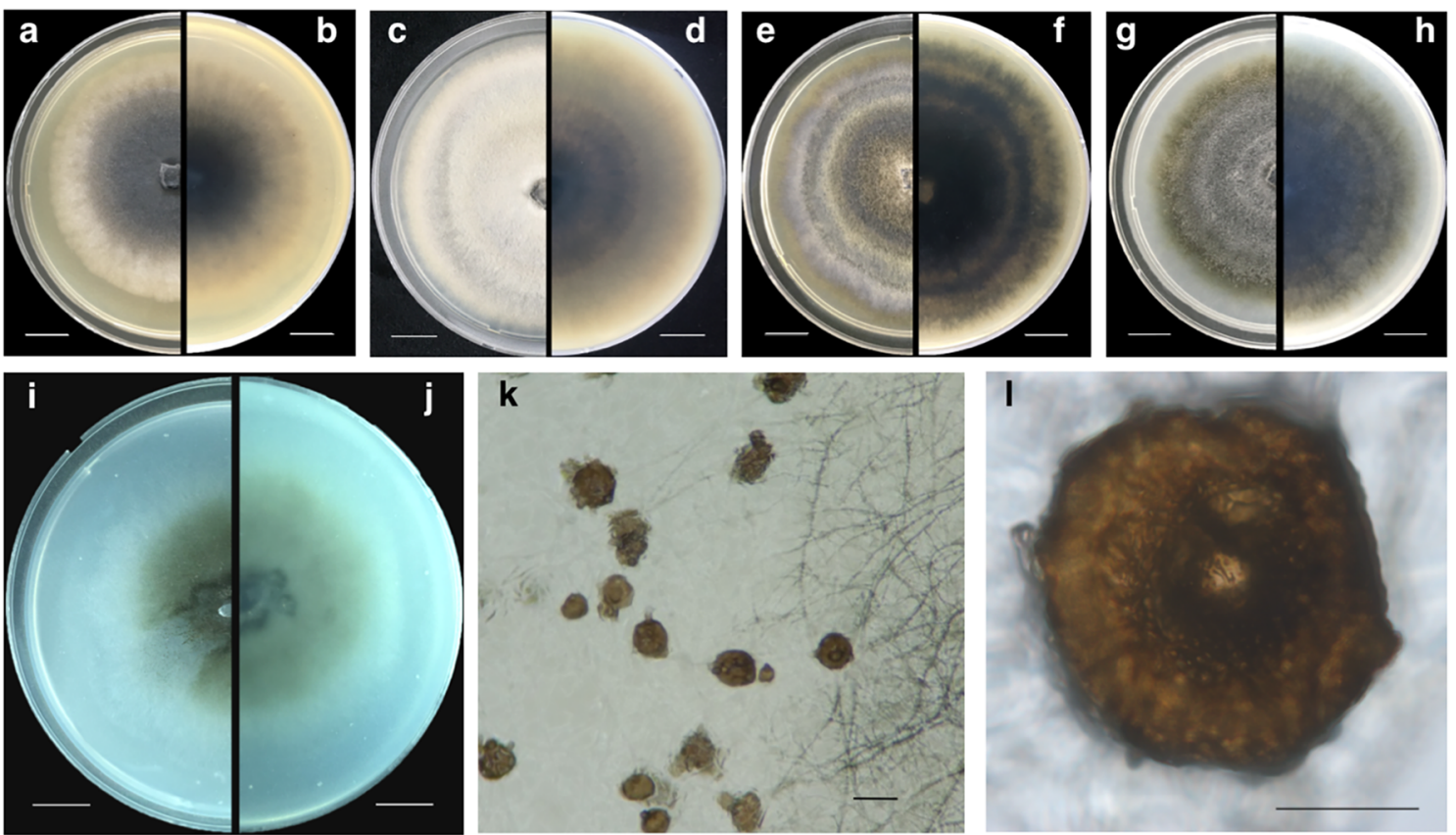

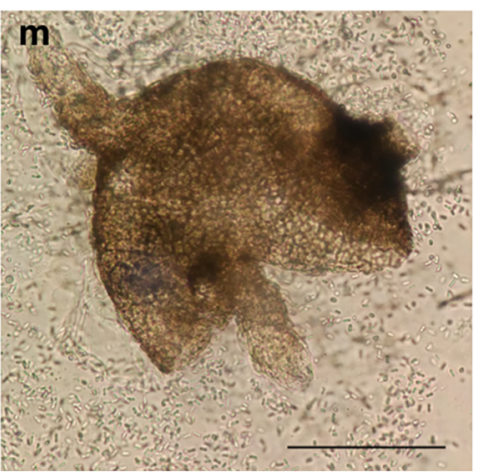

n

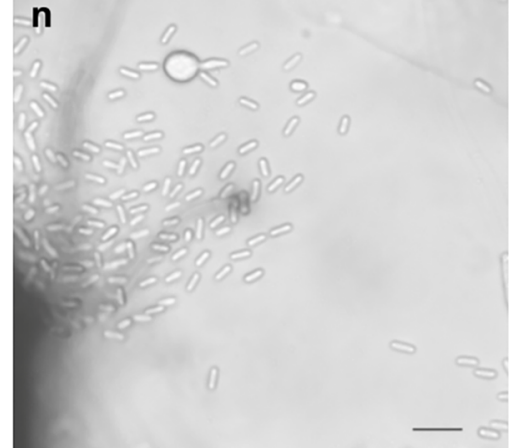

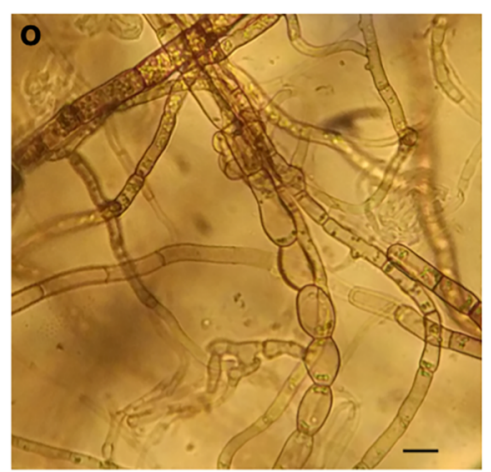

S. trachelii NJ1-2 on oatmeal agar (OA) (front and reverse) (i-j); pycnidia forming on OA (k); pycnidium (I); broken pycnidium $(\mathbf{m})$; conidia $(\mathbf{n})$; chain of chlamydospores $(\mathbf{o})$. Scale bars: $\mathrm{a}-\mathrm{j}=1 \mathrm{~cm}, \mathrm{k}=200 \mu \mathrm{m}, \mathrm{l}=$

$50 \mu \mathrm{m}, \mathrm{m}=100 \mu \mathrm{m}, \mathrm{n}-\mathrm{o}=10 \mu \mathrm{m}$ (front and reverse) (a-b), minimal salts medium (MSM) (front and reverse) (c-d), potato dextrose agar (PDA) (front and reverse) (e-f) and on V8-juice agar (V8) (front and reverse) (g-h); 10-day-old colony of 
and were globose to subglobose in shape, dark brown in color, with ostioles (Fig. 2k-1). The conidia measured 3.8-7.0 × 1.2$2.4 \mu \mathrm{m}$ (average $4.6 \times 1.8 \mu \mathrm{m}$ ) with a length-to-width ratio between 1.58 and 5.8. They were hyaline, oval to oblong, mainly aseptate, and contained small oil drops (Fig. 2n). Chlamydospores were aseptate, globose to ellipsoid, 7.4$12 \times 6-10 \mu \mathrm{m}$, light brown, thick-walled, and present in chains or clusters (Fig. 2o). These morphological characteristics were consistent with the description of Stagonosporopsis trachelii (Syn. Phoma trachelii and Ascochyta bohemica) (Garibaldi et al. 2015; Chen et al. 2015). One representative pure culture NJ1-2 was deposited in China Center of Industrial Culture Collection (CICC) with a number CICC 41704.

In addition to morphological characterisation, species identification was further validated by gene amplification of the ribosomal internal transcribed spacer (ITS) region, actin (ACT) gene region, and beta-tubulin (TUB) gene region of the 10 isolates with ITS1/ITS4 (Xiong et al. 2019), ACT512F/ACT-783R (Carbone and Kohn 1999), and DbBt-F/ DbBt-R primer pairs (Stewart et al. 2015), respectively. Multiple sequence alignment by BLASTN program revealed that the ITS, ACT or TUB sequence of the 10 isolates shared $100 \%$ sequence similarity with each other (not shown). A BLAST search in GenBank indicated three consensus sequences of isolate NJ1-2 (accession nos. MH062183.2-ITS, MK050978.1-ACT, and MK050977.1-TUB) shared 99$100 \%$ similarity with related sequences of $S$. trachelii in GenBank (strain CBS 384.68-accession no. GU237856.1, strain CBS 379.91-accession no. JN251977.1, and strain CBS 379.91-accession no. GU237678.1, respectively).

For further understanding of interspecies relationships, phylogenetic trees were constructed for the ITS, ACT and TUB sequences. The sequences used for these three phylogenetic analyses were obtained from NJ1-2, NJ3-2 and NJ4-1 isolated in this study. The ITS and TUB sequences of NJ1-2, NJ3-2 and NJ4-1 were each aligned with that of 11 other isolates of Stagonosporopsis spp. together with one isolate each of ten other fungal species (Alternaria alternata, Colletotrichum higginsianum, Corynespora cassiicola, Epicoccum sorghinum, Fusarium acutatum, Magnaporthe grisea, Phoma herbarum, Sclerotinia sclerotiorum, Trichoderma longibrachiatum, and Verticillium dahlia) obtained from the GenBank database. The same isolates of Stagonosporopsis spp. excluding one isolate of Stagonosporopsis trachelii (DB14LUG12 isolate for ITS and CBS 379.91 for TUB) were subjected to ITS- and TUB- based phylogenetic analyses (Figs. 3 and 4). For ACT-based phylogenetic analysis, a total of 23 other isolates of Stagonosporopsis spp. and one isolate each of ten other fungal species were obtained from the GenBank database (Fig. 5). A total of ten isolates each from ten

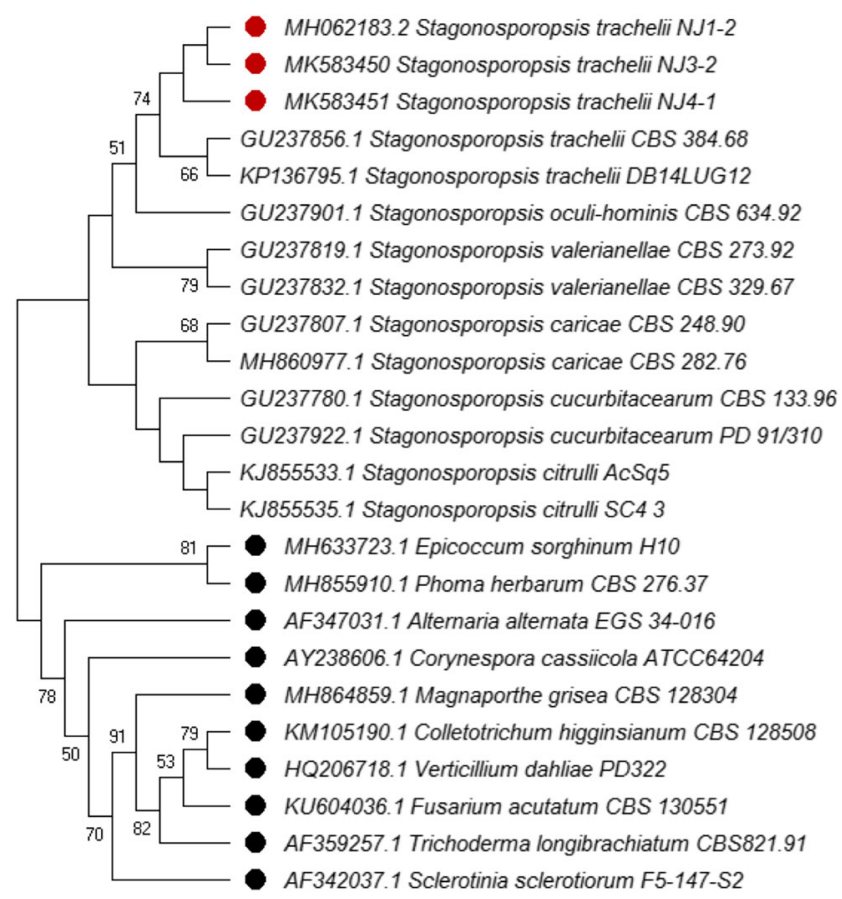

Fig. 3 Phylogenetic tree constructed with sequences of internal transcribed spacer ribosomal DNA (rDNA) region (ITS) of isolates NJ1-2, NJ3-2 and NJ4-1 obtained in this study and other species of Stagonosporopsis retrieved from GenBank. The tree was constructed using the neighbor-joining method from the alignment of ITS sequences using MEGA software (version X). Alternaria alternata, Colletotrichum higginsianum, Corynespora cassiicola, Epicoccum sorghinum, Fusarium acutatum, Magnaporthe grisea, Phoma herbarum, Sclerotinia sclerotiorum, Trichoderma longibrachiatum and Verticillium dahlia (accession nos. AF347031.1, KM105190.1, AY238606.1, MH633723.1, KU604036.1, MH864859.1, MH855910.1, AF342037.1, AF359257.1 and HQ206718.1, respectively) were used as the outgroups, which were indicated within black solid dots. Values at the nodes indicate bootstrap support values based on 1000 replicates. Bootstrap support values less than $50 \%$ are not shown. Isolates $\mathrm{NJ} 1-2, \mathrm{NJ} 3-2$ and $\mathrm{NJ} 4-1$ sequenced in this study are indicated within red solid dots

other fungal species were used as outgroups in three phylogenetic trees (Figs. 3, 4 and 5). Raw sequences were assembled with Sequencher 4.9 for Windows (Gene Codes Corp., Ann Arbor, Michigan). The consensus sequences were then initially aligned using MAFFT version 7 (Katoh et al. 2017) (http://mafft.cbrc.jp/alignment/ server/) and optimised in the SATé-II v.2.2.7 (Simultaneous Alignment and Tree Estimation) high throughput alignment platform (http://phylo.bio.ku.edu/ software/sate/sate.html) (Liu et al. 2012). Phylogenetic analysis of ITS, TUB and ACT sequence data was conducted by neighbor-joining methods using MEGA version $X$ (Kumar et al. 2018), and sequence distances were calculated based on the Maximum Composite Likelihood model. Confidence intervals were estimated using bootstrap analysis with 1000 replications. The isolates NJ12, NJ3-2 and NJ4-1 in each phylogenetic tree were placed within a monophyletic clade comprising reference 


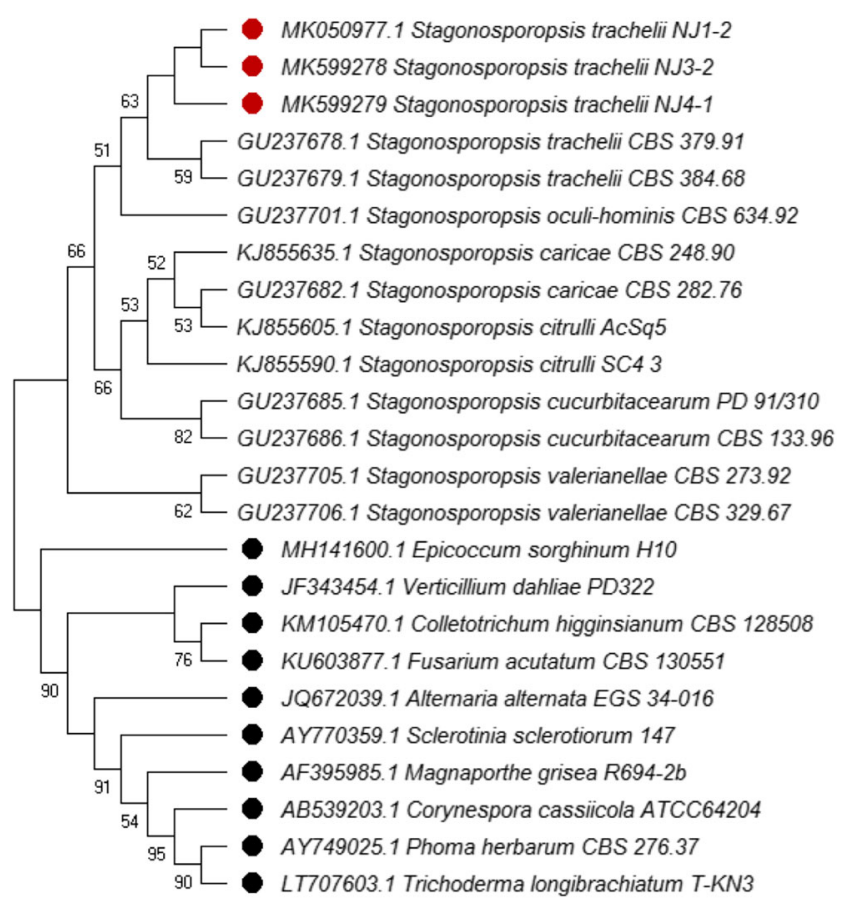

Fig. 4 Phylogenetic tree constructed with sequences of partial betatubulin of isolates NJ1-2, NJ3-2 and NJ4-1 obtained in this study and other species of Stagonosporopsis retrieved from GenBank. The tree was constructed using the neighbor-joining method from the alignment of beta-tubulin sequences using MEGA software (version X). Alternaria alternata, Colletotrichum higginsianum, Corynespora cassiicola, Epicoccum sorghinum, Fusarium acutatum, Magnaporthe grisea, Phoma herbarum, Sclerotinia sclerotiorum, Trichoderma longibrachiatum and Verticillium dahlia (accession nos. JQ672039.1, KM105470.1, AB539203.1, MH141600.1, KU603877.1, AF395985.1, AY749025.1, AY770359.1, LT707603.1 and JF343454.1, respectively) were used as the outgroups, which were indicated within black solid dots. Values at the nodes indicate bootstrap support values based on 1000 replicates. Bootstrap support values less than $50 \%$ are not shown. Isolates NJ1-2, NJ3-2 and NJ4-1 are indicated within red solid dots

isolates of $S$. trachelii species, and formed a separate clade distinct from other species of Stagonosporopsis (Figs. 3, 4 and 5). Based on molecular data, the fungus was confirmed as $S$. trachelii.

Pathogenicity assays were performed by using the drop inoculation method as described by Xiong et al. (2019). Two-week-old cultures formed on $10 \% \mathrm{~V} 8$ plates at $25{ }^{\circ} \mathrm{C}$ were flooded with sterile distilled water, and the surface was scraped. The suspension was filtered and adjusted to $10^{5}$ spores/ml with a hemocytometer. Ten healthy Ningpo figwort leaves (fifth to sixth leaf from the top in one-year-old plants) were collected and placed on $2 \%$ water agar Petri plates. Two leaves were placed in each per Petri dish, one of which was drop-inoculated with $20 \mu \mathrm{l}$ of conidial suspension of S. trachelii and the other treated with $20 \mu \mathrm{l}$ of sterile distilled water as a control. The five inoculated Petri dish plates were then sealed with Parafilm and kept in a growth chamber at $25{ }^{\circ} \mathrm{C}$ under $90 \%$ relative humidity. Typical spot symptoms,

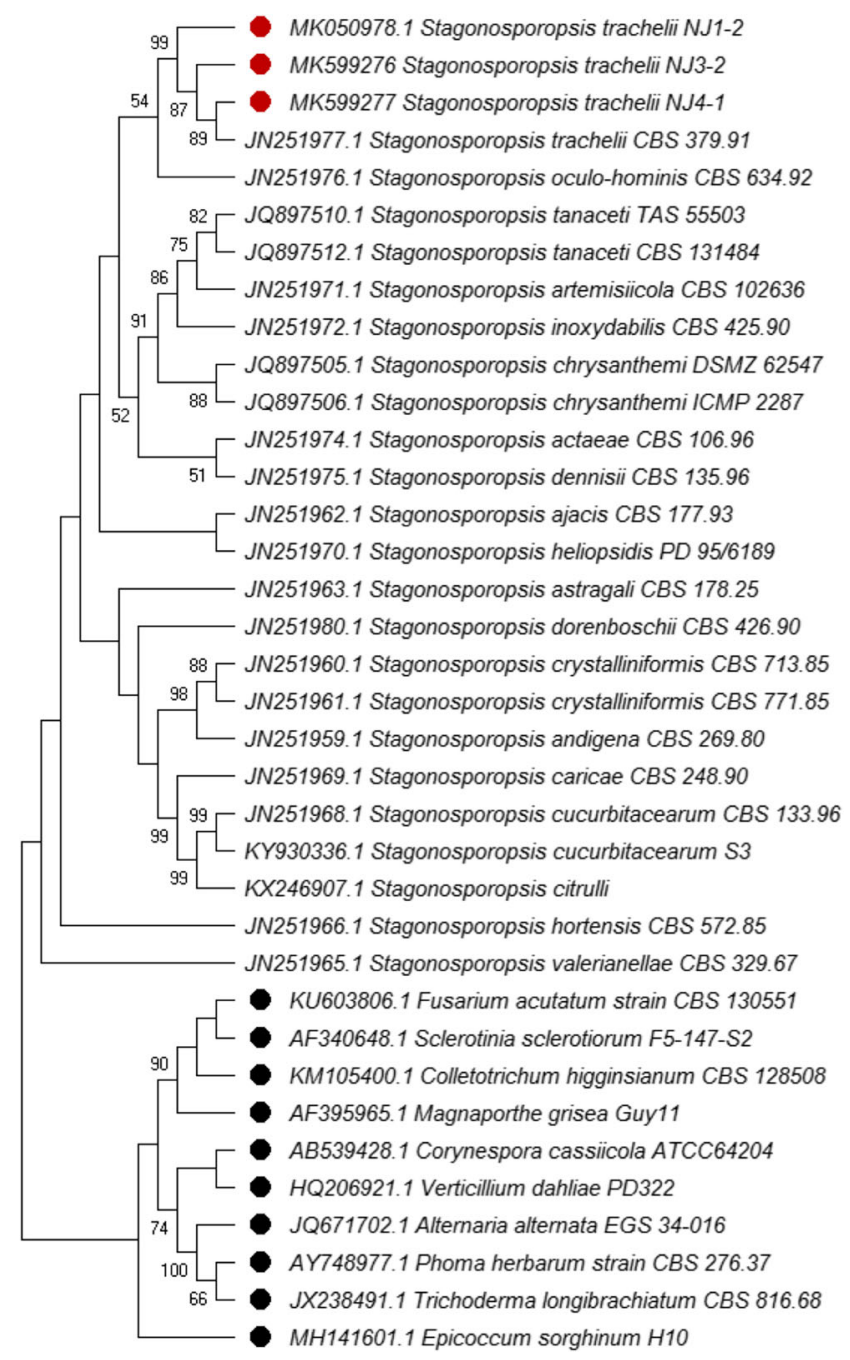

Fig. 5 Phylogenetic tree constructed with sequences of partial actin of isolates NJ1-2, NJ3-2 and NJ4-1 obtained in this study and other species of Stagonosporopsis retrieved from GenBank. The tree was constructed by neighbor-joining method from the alignment of actin gene sequences using MEGA software (version X). Alternaria alternata, Colletotrichum higginsianum, Corynespora cassiicola, Epicoccum sorghinum, Fusarium acutatum, Magnaporthe grisea, Phoma herbarum, Sclerotinia sclerotiorum, Trichoderma longibrachiatum and Verticillium dahlia (accession nos. JQ671702.1, KM105400.1, AB539428.1, MH141601.1, KU603806.1, AF395965.1, AY748977.1, AF340648.1, JX238491.1 and HQ206921.1, respectively) were used as the outgroups, which were indicated within black solid dots. Values at the nodes indicate bootstrap support values based on 1000 replicates. Bootstrap support values less than $50 \%$ are not shown. Isolates $\mathrm{NJ} 1-2, \mathrm{NJ} 3-2$ and $\mathrm{NJ} 4-1$ are indicated within red solid dots

similar to those observed in the garden, developed on all of the inoculated leaves five days after inoculation (Fig. 1d), while no symptoms developed on the control leaves. Ten days after inoculation the same fungus was re-isolated from inoculated leaves but not from controls, and the isolate was then confirmed by the morphological and molecular methods previously described, thus satisfying Koch's postulates. 
Many species of Stagonosporopsis are plant pathogens, classified in the family Didymellaceae, which cause destructive diseases to a huge range of agriculturally and ornamentally important plants, including those in forests and other natural ecosystems (Aveskamp et al. 2010). For example, Stagonosporopsis andigena and S. crystalliniformis are serious foliage pathogens on potato (Solanum tuberosum) and tomato (Solanum lycopersicum) (de Gruyter et al. 2012). Stagonosporopsis tanaceti causes ray blight of pyrethrum (Tanacetum cinerariifolium Sch. Bip.), representing one of the major biotic limitations to pyrethrum production in Australia (Chilvers et al. 2014). Stagonosporopsis chrysanthemi also causes ray blight of chrysanthemum (Chrysanthemum morifolium Ram.) and other plant species (Vaghefi et al. 2012). Three closely fungal related species of Stagonosporopsis, S. cucurbitacearum (syn. Didymella bryoniae and Phoma cucurbitacearum), S. caricae (syn. Phoma caricae-papayae), and $S$. citrulli, are major pathogens causing gummy stem blight of cucurbits worldwide and occurs everywhere they are grown, infecting at least 13 genera and 24 species of the Cucurbitaceae, including watermelon (Citrullus lanatus), cucumber (Cucumis sativus), cantaloupe (Cucumis melo var. cantalupo), muskmelon (Cucumis melo), squashes (Cucurbita pepo), and gourds (Cucurbita spp.) (Stewart et al. 2015; Brewer et al. 2015). Rennberger and Keinath (2018) recently expanded the host range of S. citrulli to 37 species representing 21 genera and seven tribes in the family Cucurbitaceae. Meanwhile, S. cucurbitacearum was recently reported as the causal agent of leaf spot on Luohanguo (Siraitia grosvenorii) (Jiang et al. 2015) and spot blight on water spinach (Ipomoea aquatica) (Liu et al. 2017) as well as tobacco (Nicotiana tabacum) (Wang et al. 2018) in China. Stagonosporopsis trachelii has only been reported to cause leaf spot on Campanula medium in Italy (Garibaldi et al. 2015). To our knowledge, this is the first report of S. trachelii causing leaf spot on $S$. ningpoensis in China. Further studies are needed to determine the distribution and to assess the economic impacts of this disease in China.

Acknowledgements This study was supported by the National Natural Science Foundation of China (grant no. 31600512), the Natural Science Foundation of Jiangsu Province (grant no. BK20160923), Students Practice Innovation and Training Program of Nanjing Forestry University (201710298045Z, 201710298064Z, 2016NFUSPITP206), China Scholarship Council (CSC), and the Priority Academic Program Development of Jiangsu Higher Education Institutions.

\section{Compliance with ethical standards}

Conflict of interest The authors declare that they have no conflict of interest.

Human and animals participants This article does not contain any studies with human participants or animals performed by any of the authors.

\section{References}

Aveskamp MM, de Gruyter J, Woudenberg JHC, Verkley GJM, Crous PW (2010) Highlights of the Didymellaceae: a polyphasic approach to characterize Phoma and related pleosporalean genera. Stud Mycol 65:1-60. https://doi.org/10.3114/sim.2010.65.01

Brewer MT, Rath M, Li H-X (2015) Genetic diversity and population structure of cucurbit gummy stem blight fungi based on microsatellite markers. Phytopathology 105(6):815-824. https://doi.org/10. 1094/PHYTO-10-14-0282-R

Carbone I, Kohn LM (1999) A method for designing primer sets for speciation studies in filamentous ascomycetes. Mycologia 91(3): 553-556. https://doi.org/10.2307/3761358

Chen Q, Jiang JR, Zhang GZ, Cai L, Crous PW (2015) Resolving the Phoma enigma. Studs Mycol 82:137-217. https://doi.org/10.1016/j. simyco.2015.10.003

Chilvers MI, Jones S, Meleca J, Peever TL, Pethybridge SJ, Hay FS (2014) Characterization of mating type genes supports the hypothesis that Stagonosporopsis chrysanthemi is homothallic and provides evidence that Stagonosporopsis tanaceti is heterothallic. Curr Genet 60(4):295-302. https://doi.org/10.1007/s00294-014-0435-0

de Gruyter J, van Gent-Pelzer MP, Woudenberg JH, van Rijswick PC, Meekes ET, Crous PW, Bonants PJ (2012) The development of a validated real-time (TaqMan) PCR for detection of Stagonosporopsis andigena and S. crystalliniformis in infected leaves of potato and tomato. Eur J Plant Pathol 134(2):301-313. https://doi.org/10.1007/s10658-012-9990-8

Garibaldi A, Bertetti D, Ortu G, Gullino M (2015) A leaf spot caused by Stagonosporopsis trachelii on Campanula medium in Italy. J Plant Pathol 97(3):546. https://www.jstor.org/stable/24722566. Accessed 01 July 2015

Jiang N, Hu F, Ye Y, Jiang SY, Huang X (2015) First report of leaf spot caused by Stagonosporopsis cucurbitacearum on Luohanguo (Siraitia grosvenorii) in China. Plant Dis 99(11):1645. https://doi. org/10.1094/PDIS-12-14-1259-PDN

Katoh K, Rozewicki J, Yamada KD (2017) MAFFT online service: multiple sequence alignment, interactive sequence choice and visualization. Brief Bioinform. https://doi.org/10.1093/bib/bbx108

Kumar S, Stecher G, Li M, Knyaz C, Tamura K (2018) MEGA X: molecular evolutionary genetics analysis across computing platforms. Mol Biol Evol 35(6):1547-1549. https://doi.org/10.1093/molbev/ msy096

Li YM, Jiang SH, Gao WY, Zhu DY (1999) Iridoid glycosides from Scrophularia ningpoensis. Phytochemistry 50(1):101-104. https:// doi.org/10.1016/S0031-9422(98)00477-4

Li J, Huang X, Du X, Sun W, Zhang Y (2009) Study of chemical composition and antimicrobial activity of leaves and roots of Scrophularia ningpoensis. Nat Prod Res 23(8):775-780. https:// doi.org/10.1080/14786410802696247

Liu K, Warnow TJ, Holder MT, Nelesen SM, Yu J, Stamatakis AP, Linder CR (2012) SATé-II: very fast and accurate simultaneous estimation of multiple sequence alignments and phylogenetic trees. Syst Biol 61(1):90. https://doi.org/10.1093/sysbio/syr095

Liu P, Wei M, Zhu L, Li B, Weng Q, Chen Q (2017) First report of spot blight on water spinach (Ipomoea aquatica) caused by Stagonosporopsis cucurbitacearum in China. Plant Dis 101(5): 838. https://doi.org/10.1094/PDIS-10-16-1485-PDN

Pasdaran A, Delazar A, Nazemiyeh H, Nahar L, Sarker SD (2012) Chemical composition, and antibacterial (against Staphylococcus aureus) and free-radical-scavenging activities of the essential oil of Scrophularia amplexicaulis Benth. Rec Nat Prod 6(4):350-355

Rennberger G, Keinath AP (2018) Susceptibility of fourteen new cucurbit species to gummy stem blight caused by Stagonosporopsis citrulli 
under field conditions. Plant Dis 102(7):1365-1375. https://doi.org/ 10.1094/PDIS-12-17-1953-RE

Stewart JE, Turner AN, Brewer MT (2015) Evolutionary history and variation in host range of three Stagonosporopsis species causing gummy stem blight of cucurbits. Fungal Biol 119(5):370-382. https://doi.org/10.1016/j.funbio.2014.12.008

Vaghefi N, Pethybridge SJ, Ford R, Nicolas ME, Crous PW, Taylor PWJ (2012) Stagonosporopsis spp. associated with ray blight disease of Asteraceae. Australas Plant Path 41(6):675-686. https://doi.org/10. 1007/s13313-012-0161-3

Wang C, Zhou H, Yu Z, Zhang Z, Chen X, Cai L (2018) Spot blight on tobacco (Nicotiana tabacum L.) caused by Stagonosporopsis cucurbitacearum in Guizhou Province of China. Plant Dis 102(10):2030. https://doi.org/10.1094/PDIS-11-17-1793-PDN

Xiong Q, Qian Y, Zhang C, Shi N, Zheng X (2019) First report of Phytophthora hydropathica causing wilting and shoot blight on Bixa orellana in China. Plant Dis 103(1):163. https://doi.org/10. 1094/PDIS-06-18-1013-PDN

Yang S, Li J, Zhao Y, Chen B, Fu C (2011) Harpagoside variation is positively correlated with temperature in Scrophularia ningpoensis Hemsl. J Agric Food Chem 59(5):1612-1621. https://doi.org/10. $1021 /$ jf104702u 